\title{
MODELAGEM E TENDÊNCIA DA TEMPERATURA MÉDIA DO AR DA CIDADE DE SALVADOR-BAHIA
}

Alexandre B. Lopo ${ }^{1}$, Maria Helena C. Spyrides ${ }^{1}$, Paulo S. Lucio ${ }^{1}$, Javier Sigró ${ }^{2}$ e Washington L. F. Correia Filho ${ }^{1}$

${ }^{1}$ Programa de Pós-Graduação em Ciências Climáticas-UFRN.

${ }^{2}$ Universidade Rovira i Virgili (Tarragona, Espanha).

\section{RESUMO}

O presente trabalho verifica a tendência e realiza a modelagem da Temperatura média do ar (Tma) da cidade de Salvador (BA), com dados de 1961 a 2012, visando a projeção de sua variabilidade para um período climatológico de 30 anos (2013-2042). O teste de MannKendall indicou tendência positiva em Tma e o Modelo de Regressão Dinâmica ajustado estimou uma Tma de $25,8^{\circ} \mathrm{C}$, acima da atual Normal climatológica de $25,3^{\circ} \mathrm{C}$.

\begin{abstract}
This paper verifies the trend and performs the modeling of the mean air temperature (Tma) Salvador (BA) city, with data from 1961 to 2012 with a view to projecting its climatological variability for a period of 30 years (2013-2042). The trend test of Mann-Kendall indicated positive trend in Tma and Dynamic Regression Model estimated an Tma of $25.8^{\circ} \mathrm{C}$ above the climatological normal current of $25.3^{\circ} \mathrm{C}$.
\end{abstract}

\section{INTRODUÇÃO}

No atual contexto de aquecimento global, apontado pelo $4^{\circ}$ Relatório do Painel Intergovernamental de Mudanças Climáticas (IPCC-AR4/ONU), torna-se relevante analisar e modelar a Temperatura média do ar (Tma) visando identificar os possíveis sinais ou evidências de tendências das mudanças climáticas nos grandes centros populacionais, neste caso abordamos a cidade de Salvador (Bahia). Metodos estatísticos para caracterizar estas alterações vem sendo implementados em clima, neste trabalho utilizamos o Modelo de Regressão Dinâmica (MRD) (PANKRATZ, 1991), que combina os valores históricos da 
série temporal e variáveis regressoras, ambas com ou sem defasagens. O objetivo deste trabalho será modelar a Tma pelo modelo de regressão dinâmica para os próximos 30 anos (2013-2042) e verificar sua tendência.

\section{MATERIAIS E MÉTODOS}

Os dados de Tma e UR são do período de 1961 a 2012 do INMET, oriundas da cidade de Salvador-Bahia e disponibilizados através do Banco de Dados Meteorológicos para Ensino e Pesquisa (BDMEP) em www.inmet.gov.br. O Teste Mann-Kendall foi aplicado nesses dados para analisar a tendência de variação na intensidade e quantidade de eventos.

A modelagem via MRD consiste de uma regressão envolvendo séries temporais que inclui valores atuais e passados da variável em estudo e variáveis regressoras, ambas com ou sem defasagens (lag) (PANKRATZ, 1991). Esse modelo (Equação 1) é conhecido como modelo de defasagens distribuídas autoregressivo, $\operatorname{ADL}\left(\mathrm{p}_{\mathrm{j}}, \mathrm{p}\right)$, onde, os $\mathrm{p}_{\mathrm{j}}$ e $\mathrm{p}$ indicam a ordem de defasagem das variáveis ou variável, respectivamente, dependente e independente.

$$
\mathrm{y}_{\mathrm{t}}=\alpha+\sum_{\mathrm{j}=1}^{\mathrm{k}} \sum_{\mathrm{i}=1}^{\mathrm{q}_{\mathrm{j}}} \beta_{\mathrm{ji}} \mathrm{x}_{\mathrm{jt}-\mathrm{i}}+\sum_{\mathrm{i}=1}^{\mathrm{p}} \emptyset_{\mathrm{i}} \mathrm{y}_{\mathrm{t}-\mathrm{i}}+\varepsilon_{\mathrm{t}}
$$

Em que: $\mathrm{y}_{\mathrm{t}}$ : variável dependente em $\mathrm{t} ; \alpha$ : constante; $\mathrm{y}_{\mathrm{t}-\mathrm{i}}$ : variável dependente em $\mathrm{t}-\mathrm{i} ; \mathrm{x}_{\mathrm{jt}-\mathrm{i}}: \mathrm{j}$ ésima variável independente em $\mathrm{t}-\mathrm{i}$, sendo $\mathrm{i}=\left\{1, \ldots, \mathrm{p}_{\mathrm{j}}\right\}$ e $\mathrm{j}=\{1, \ldots, \mathrm{p}\} ; \beta_{\mathrm{ji}}$ : coeficiente da $\mathrm{j}$-ésima variável independente em t-i; $\varphi_{\mathrm{i}}$ : coeficiente da variável dependente em t-i;e $\varepsilon_{\mathrm{t}}$ : erro aleatório.

Para estimar $\beta_{0}$ e $\beta_{l}$, utilizaram-se os dados diários de Tma e como variável explicativa a UR. Para testar a significância (5\%) da equação de regressão, realizou-se o teste $F$ e para os coeficientes o teste $t$-Student. (NETER et al., 1996).

A aplicação do MRD pressupõe a verificação de pressupostos associados aos erros $\left(\varepsilon_{t}\right)$ : os erros são variáveis aleatórias de média zero e variância constante $\left(\sigma^{2}\right)$-hipótese de homocedasticidade; as variáveis aleatórias $\varepsilon_{1}, \varepsilon_{2}, \ldots ., \varepsilon_{n}$ são independentes e seguem uma distribuição normal: $\varepsilon_{1} \sim \mathrm{N}\left(0, \sigma^{2}\right)$. O teste Breusch-Pagan (BREUSCH \& PAGAN, 1979) foi utilizado para identificar se os erros eram homocedásticos ou heterocedásticos e o teste Kolmogorov-Smirnov (KS) para verificar a normalidade. Além dos erros, testa-se nas variáveis explicativas $x_{1}, x_{2}, \ldots, x_{k}$ a hipótese de ausência de multicolinearidade através do 
Fator de Inflação da Variância (VIF). Não aplicado nesse estudo devido a utilização de apenas uma variável explicativa.

A estimativa dos parâmetros ou coeficientes de regressão da equação ocorreu utilizando o método de Mínimos Quadrados Ordinários e os resultados foram obtidos através dos procedimentos realizados por meio do software R, pacote dynlm (ZEILEIS, 2012).

\section{RESULTADOS E DISCUSSÃO}

Iniciou-se a modelagem por meio da Função Correlação Cruzada (CCF), utilizada para mensurar as defasagens temporais entre a variável resposta e a variável explicativa, cujo resultado mostrou defasagens de maior correlação para 6 e 12 meses ( $\operatorname{lag}_{6}$ e $\operatorname{lag}_{12}$ ) em referência a própria variável resposta. A UR não apresentou defasagem com correlação significativa. A Tabela 1 apresenta os parâmetros do modelo final.

Tabela 1 - Estimação dos coeficientes/defasagens e valor-p do modelo.

\begin{tabular}{l|l|l|l}
\hline Variáveis & Coeficiente/defasagens & Erro & valor-p \\
\hline intercepto $_{\mathrm{t}}$ & 15,75 & 1,51 & $<0,001$ \\
${\text { (ur })_{\mathrm{t}}}_{\text {tma }_{\mathrm{t}}}$ & $-0,04$ & 0,01 & $<0,001$ \\
(tma $_{\mathrm{t}}$ & $-0,21_{(\operatorname{lag} 6)}$ & 0,03 & $<0,001$ \\
& $0,72_{(\operatorname{lag} 12)}$ & 0,03 & $<0,001$ \\
\hline
\end{tabular}

A equação de estimação dos parâmetros do modelo é expressa:

Tma =15,75 - 0,04 ur -0,21tma (lag6) $+0,72 t m a(\operatorname{lag} 12)+e_{t}(1)$

Pelo teste $F$, a equação de regressão apresentou significância estatística (valor-p <0,05) e os coeficientes de forma similar (valor-p na tabela refere-se ao teste t-Student. A, normalidade dos erros foi confirmada pelo teste KS (valor-p=0,48). Já pelo teste Breusch-Pagan foi constatado o valor-p de 0,19 , sendo p $>0.05$ confirmando a hipótese de homocedasticidade dos resíduos.

Com o modelo (Equação 1), realizou-se a predição (interpolação) dos dados e aferiu-se a qualidade e validade via análise de correlação e cálculo do erro quadrático médio (MSE) entre observações e dados interpolados. Os resultados apontaram um coeficiente de correlação de 0,88 e MSE $=0,43$.

A previsão estimada (extrapolação) da Tma para o período de 30 anos (2013-2042) indicou que a média climatológica será de $25,6 \pm 0,1^{\circ} \mathrm{C}$, e para o ano 2042 esta média aponta o valor 
de $25,8 \pm 0,8^{\circ} \mathrm{C}$, ambas acima da Normal climatológica atual de $25,3^{\circ} \mathrm{C}$ definida pelo INMET para o período de 1961-1990.

O teste Mann-Kendall foi significativo (valor-p<0,05) e confirmou a tendência positiva ou de elevação $(Z=0,385)$ da Tma no período de 1961-2012.

\section{CONCLUSÕES}

O modelo constituído estimou um elevação para a Tma na cidade de Salvador nos próximos 30 anos (2013-2042). A perspectiva para trabalhos futuros será inserir outras variáveis explicativas para aperfeiçoamento do modelo.

\section{AGRADECIMENTOS}

O presente trabalho foi realizado com apoio do CNPq- Brasil. O co-autor Washington. L. F. Correia Filho agradece a CAPES pela bolsa de doutorado.

\section{REFERÊNCIAS}

BREUSCH, T.; PAGAN, A. A Simple Test of Heteroscedasticity and Random Coefficient Variation. Econometrica, 47, p. 1287-1294, 1979.

NETER, J.; KUTNER, M. N.; NACHTSSHEIM, C. J.; WASSERMAN, W. Applied linear statistical models. Boston:WCB/McGraw-Hill, 4ª Ed., p. 791, 1996.

PANKRATZ, A. Forecasting with dynamic regression models. John Wiley and Sons, New York, 1991.

ZEILEIS, A. 2012. dynlm: Dynamic Linear Regression. R package version 0.3, 2012. Disponível em:<http://cran.r-project.org/package=dynlm>.Acesso em: 10 maio. 2013. 